XVI.

Aus der Frauenklinik von Prof. L. Fraenkel-Breslau.

\title{
Ueber Erblichkeit der Ovarial-, besonders der Dermoidzysten.
}

\author{
Von \\ Dr. Hermann Koltonski, \\ Oberarat der Klinik.
}

In Pfannenstiel's Abhandlung über „die Erkrankungen des Eierstocks und Nebeneierstocks" (Veit's Handbuch der Gynäkologie) findet sich folgender Passus: Ueber die hereditäre Veranlagung zu Ovarialgeschwülsten wissen wir noch wenig. Die Zahl der Fälle von Ovarialgeschwülsten bei Schwestern oder in anderer. Weise Blutsverwandten ist sogar auffallend gering im Verhältnis zu der Hänfigkeit der Eierstocksneubildungen im allgemeinen.

Diese Worte lassen es berechtigt erscheinen, uiber folgende Fälle, die in der L. Fraenkel'schen Klinik zur Beobachtung gelangt sind, zu referieren.

Es handelt sich um 5 Patientimmen, die zwei rerschiedenen Familienkreisen angehören.

1. F. K., 44 J., Jalirgang 1901. Anamnese: Seit dem 18. Lebensjahre dauernd kränkelnd; die Klagen bezogen sich hauptsächlich auf krampfartige Scbmerzen im linken Hypogastrium, die mitunter von anßerordentlicher Heftigkeit waren und als "nervös" gedeutet wurden. Die erste gynäkologische Untersuchung erfolgte erst kurz vor der später zu schildernden Operation. Vater und drei Schbwestern - von im ganzen 5 Geschwistern - sind bis zum Jahre 1919 an Karzinom des Verdauungstraktus gestorben. Erste Menses mit 14 Jahren, regulär, 4wöchentlich, schwach. November 1900 „Darmverschlingung" (Stieldrehung des Tumors?). 3 Partus, o. B., zuletzt vor 11 Jahren.

Status (Juli 1901). Untermittelgroße, grazil gebaute Frau, mäßige Muskulatur, geringer Pannikulus, anämisch-kachektisches Aussehen. Innere Organe gesund. Vulva klaffend. Descensus vaginae post. mit hühnereigroßer Rektozele. Dammriss I. Uteruskörper ein wenig retrovertiert, dicht dem Kreuzbein aufliegend. Vor dem Uterus ein etwas beweglicher, weicher Tumor, von der Gröbe einer Mannsfaust und daneben ein hühnereigroßer, oblonger Tumor, deutlich mit beiden Fundalecken durch bleifederdicke, drehrunde Stränge zusammenhängend. Juli 
1901 wird per laparotomiam der Tumor samt den beiderseitigen Adnexen exstirpiert; es bestanden umfangreiche Adbäsionen zwischen dem Tumor einerseits, Netz, Blasenwand und Darmteilen andererseits.

Bescbreibung des Präparats: Dieses besteht aus den beiden in der Ausdehnung eines Pfennigstücks fest miteinander verlöteten Ovarien. Das rechte ist gänseeigroß, das linke mannsfaustgrob. Letzteres ist auf der Oberfläche mit Psendomembranen und Netzgewebe bedeckt und besteht im wesentlichen aus mehreren bis walnußgrossen Zysten. Die eine ist sehr dünnwandig, geplatzt, die andere mit derber Wand und hellerem Inhalt. Die linksseitige ist eine Dermoidzyste mit Haaren, Brei, Zähnen und einer Dermoidzotte. Letztere in einem besonderen Abteil der sonst einkammerigen Zyste.

Acht Monate später (Februar 1902) sucht die einzige Tochter der Patientin die Klinik auf:

G. L., 19 Jahre alt, erste Menses mit etwa 12 Jahren, regulär, 4 tägig stark. Seit 3 Jahren Dysmenorrhoe. - Vier Monate verheiratet. Fehlgeburt am 4. Januar 1902, (letzte Menstruation 14. Oktober 1901), spontan, ohne starke Blutung, ohne Fieber.

Keine Beschwerdeñ, kommt nur wegen des Abortes.

Status: Etwas unter Mittelgröße, grazil, geringer Pannikulus, innere Organe gesund. - Portio steht normal, einpfennigstückgroße Erosion. Uterus anteflektiert, klein, beweglich, links hinter ihm orangengroßer, praller Tumor, etwas beweglich, rund, dünnwandig, mit der linken Fundalecke zusammenbängend; liegt eingepreßt im Douglas. Rechte Adnexe hühnereigroß, rechts hinter dem Uterus liegend, wenig beweglich, ziemlich hart, anscheinend adhärent.

Operation (19. Februar). In Chloroformäthernarkose Medianschnitt; 3 EBlöffel freier Aszites im Abdomen. Links hinter dem Uterus wird faustgrobe Zyste leicht hervorgeholt und exstirpiert. Tube gesund, wird zurückgelassen. Rechtes Ovarium hühnereigroß, nicht adhärent, platt. Mediale Hälfte normal, laterale Hälfte in eine walnußgroße Zyste verwandeit. Diese wird ignipunktiert.

Beschreibung des Präparats: Fast mannskopfgroße Zyste mit graubläulicher Wand und glatter Oberfläche. Aufgeschnitten entleert sich öliger Brei mit schwarzen, kurzen Haaren, ferner enthält er eine etwa $4 \mathrm{~cm}$ lange Knorpelknochenleiste, welcher 2 Zühne aufsitzen. Auf. der Oberflüche der Zyste finden sich noch eine Anzahl halbreifer Follikel, sowie ein zweipfennigstückgroßes, $1 / 2 \mathrm{~cm}$ dickes (also plattgodrücktes) Corpus lutenm, offenbar von der vorangegangenen Gravidität herrührend.

Hutter. und Tochter sind also an rechtsseitigen Dermoidzysten erkrankt, die linken Ovarien sind ebenfalls zystisch entartet. Die Aehnlichkeit der Fälle springt stark in die Augen, und der Gedanke der Heredität drängt sich einem-naturgemäß von selbst auf.

Die Dermoide und Teratome haben in der Reihe der Geschwulstbildungen wegen ihres eigenartigen Charakters schon immer eine Sonderstellung eingenommen, die sich auch auf ihre Genese erstreckte, derart, daß Olshausen sogar soweit geht und die Entstehungsweise der Der- 


\section{Koltonski, Ueber Erblichkeit der Ovarial-, besonders der Dermoidzysten.}

moide gesondert von der Aetiologie der anderen Genitaltumoren behandelt. Vielleicht weil ihm das Werden dieser Geschwülste wegen ihrer Herkunft von allen 3 Keimblättern eine besondere Betrachtung notwendig erscheinen ließ, vielleicht aber auch, weil sich bei Dermoidzysten das hereditäre Moment ihm besonders manifestierte. Denn mehrere Autoren - ich erwähne Simpson, Rose, Lever, Boivin - berichten von Fällen, in denen mehrere Schwestern an Ovarialzysten erkrankten, und Olshausen selbst operierte 3 Schwesternpaare. Der Gedanke der Heredität erschien auch deswegen naheliegend, weil die Dermoide in allen Lebensaltern vorkommen: "Kein Alter schließt die Entstehung von Ovarialkystomen der einen oder anderen Art aus, nur vom fötalen Alter liegen Beobachtungen noch nicht vor" - meint Olshausen; aber auch letztere Einschränkung muß fallen gelassen werden, nachdem v. Winckel uns die Seltenheit einer doppelseitig angeborenen Eierstocksgeschwulst beschrieben hat. Das Präparat stammte von einer Frühgeburt, die 3 Tage post partum starb. Uebrigens berichtete Christel vor kurzem von 2 Dermoiden, die er an einem 15- und 5jährigen Mädchen zu entfernen genötigt war, und Römer mußte sogar einem 20monatigen Kinde ein Dermoidkystom, das bis zur Mitte zwischen Nabel und Schwertfortsatz reichte, exstirpieren.

Auch in unserem geschilderten Falle ist der Gedanke der Heredität ein durchaus naheliegender; zwar könnte der Einwand erhoben werden, hier läge ein Zufall vor insofern, als die mütterliche Patientin am Tumor erst im 41. Lebensjahre erkrankt sei, also in einem Alter, in dem sich schon, abgesehen von der langen Zeitspanne selbst, reichlich Gelegenheit zur Geschwulstbildung geboten hat durch Generationsvorgänge, Traumen, entzünd́liche Prozesse usw.; doch wäre hierbei zu berücksichtigen, daß es zunächst durchaus wahrscheinlich erscheint,' daß der Tumor (vielleicht sogar in gleicher Größe) schon seit dem 18. Lebensjahre bestand, also bereits zur Pubertätszeit (vgl. Anamnese, die heftigen Beschwerden, die Pat. seitdem zu haben angibt); es ist aber auch bekannt, daß Dermoide oft viele Jahre lang bestehen können - zum mindesten in ihrer Anlage ohne die geringsten subjektiven, mitunter auch objektiven Symptome zu verursachen. Hierbei wäre noch gar nicht die rein begriffliche Frage der Heredität berücksichtigt, eine Frage, die hier nur andeutungsweise gestreift werden kann: Wird nämlich die Geschwulst als solche übertragen, d. h. gehen Tumorzellen vom mütterlichen Organismus auf den Fötus nach Art einer Metastase über, oder wird nur Disposition vererbt, d. h. die erbliche Uebertragung der Neigung zu Entwickelungsstörüngen, örtlichen Gewebsmißbildungen, Keimversprengungen usw.? Durch solche Ereignisse würden in einem Gewebsbezirk oder im Bereiche eines ganzen Systems Bedingungen geschaffen, welche, unter Mitwirkung von Gelegenheitsursachen, die Grundlage zu einem blastomatösen Wachstum werden können. - 
Koltonski, Ueber Erblichkeit der Ovarial-, besonders der Dermoidzysten. 411

Schlössen wir uns der Theorie der vererbten Disposition an, dann hätte das Manifestwerden der Dermoidzyste erst im 41. Jahr nichts Befremdliches mehr; dann bekäme aber auch die Familienanamnese in vorliegendem Falle eine erhöhte Bedeutung für uns: In drei Generationen sind nicht weniger als bisher 6 Tumorfälle manifest geworden - 4 mal Karzinom, 2 mal Dermoidzysten - ein Geschehnis, bei dem es einem schwer fällt, von einem Zufall zu sprechen. Bevor ich des Näheren hierauf eingehe, lasse ich zünächst einen Bericht über folgende 3 Fälle - Mutter nebst 2 Töchtern - folgen:

1. E. Th., Jahrg. 1913, 41 Jahre. - Familienanamnese o. B. Menstruation regelmäßig, 4 wöchentlich, seit 1 Jahr 2-3 wöchentlich von 8-12tägiger Dauer. Verheiratet seit dem 21. Jahr. 4 Partus, zuletzt 1904 , nie gestillt. Seit 2 Jahren klagt sie über "Druck nach unten und auf den Mastdarm“.

Mittelgroße, kräftig gebaute Frau. Abdomen weich, nicht aufgetrieben. Bimanuell: Uterus zwischen gänseei- und kleinkindskopfgroß, frei beweglich. Adnexe nicht sicher durchzutasten. Im August 1913 wird auf vaginalem Wege das Corpus uteri amputiert. Es ist über. gänseeigroß, von glatter Oberfläche und weicher Konsistenz. Nach dem Aufschneiden sieht man, von der Hinterwand ausgehend, ein gänseeigroßes, subseröses Myom, breitbasig aufsitzend. - Glatter Verlauf.

2 Monate vorher kam deren damals 15 Jahre alte Toobter

2. E. Th. ebenfalls zur Operation.

Früher gesund, seit längerer Zeit Kreuzschmerzen. 1. Menses vor 6 Monaten, dann 3 Monate Amenorrboe, darauf wieder 1 mal Menses vor 5 Wochen. Nach 2 Wochen wieder Blutung mit sehr heftigen Leibschmerzen, die bis zur Aufnahme anhalten.

Mittelgrobes, chlorotisches Mädchen. Innere Organe gesund.

Bimanuell: Vor dem kleinen, anteflektierten Uterus liegt eine mannsfaustgrobe, zystische Geschwulst, die mit einem Stiel zur Uteruskante zu reichen scheint. - Dureh Laparotomie wird im Juni 1913 der Tumor, eine 2 mal um seine Achse gedrehte, rechtsseitige Dermoidzyste, samt Tube exstirpiert. - Das Präparat ist mannskopfgroß, teils bläulich, teils weiß durchschimmernd von wechselnder Konsistenz. Ueber ihn zieht die verdickte Tube, deren Trichter geöffnet ist. Beim Aufschneiden erweist sich der größte Teil des Tumors als Zyste mit serösem Inhalt, nur an einer Stelle ist etwas Dermoidbrei und eine Zotte.

Verlauf: Leichte Bronchitis, sonst glatt.

Im Jahr 1919 wurde die zweite Tochter

3. H. Th., $17^{1} / 4$ Jahre alt, in unserer Klinik operiert.

Mittelgroßes, grazil gebautes Mädchen. Befriedigender Ernährungszustand. Klagt über Rücken- und Magenschmerzen seit 1 Jahr.

Hymen ist intakt. Bimanuell fühlt man einen Tumor, der fast bis zum Nabel heraufreicht; er ist kugelig und prall gespannt. Auch in Narkose läßt sich der Uterus, nachdem der Tumor hoch nach oben abgedrängt ist, nicht gesondert abtasten. - Im März 1919 wird Laparotomie ausgeführt und der kindskopfgrobe, 
intraligamentär entwickelte Tumor - nach Punktion - samt T'ube entfernt. Die rechten Adnexe sind gesund. Glatter Verlauf. Präparat: Der Tumor ist kindskopfgroß, prall, bläulich durchscheinend. Nach der Eröffnung entleert sich noch etwa 1 Liter seröser klarer Flüssigkeit, so daß der Tumor im ganzen etwa $11 / 2$ Liter enihalten hatte. Die Innenwand der Zyste ist glatt und spiegelnd. Gefäßzeichnung sebr ausgesprochen. An der Oberfläche ein Gebilde von Mandelgröße, das der Ovarialrest zu sein scheint. An der Innenfläche ziehen 4 Wülste von $6-8 \mathrm{~cm}$ Länge hin, die sich allmählich in der Tumorwand verlieren. Aus dem Wulst werden Schnitte entnommen und mikroskopiert, jedoch nur gewöhnliche Zystenwand festgestellt, ohne Dermoidgebilde. Das mandelförmige Gebilde erweist sich mikroskopisch als Ovarium. Der Tumor wird als Parovarialzyste diagnostiziert.

Hulter und 2 Töchter sind also an 3 - genetisch verschiedenen Tumorarten erkrankt ${ }^{1}$ ). In diésen 3 Fällen kann man natürlich von einem zufälligen Zusammentreffen nicht sprechen, und es wird nur übrig bleiben anzunehmen, $\mathrm{da} \beta$ in der Familie Disposition zur Tumorbildung in allgemeinen herrscht.

Es erscheint mir an dieser Stelle notwendig, die Genese dieser 3 Tumorarten - gemäß -dem augenblicklichen Stande der Wissenschaft - ganz kurz zu streifen: Einheitlichkeit der Ansichten über die Entstehung der Myome gibt es bisher noch nicht; v. Recklinghausen nimmt auf Grund des eigentümlichen Befundes von Drüsen in manchen Myomen an, daß es sich um Wucherung angeborener oder doch in der Entwicklung des Genitalschlauches angelegter Keime handelt. (Als Quellen der drüsigen Einlagerungen kämen in Betracht entweder Reste des Wolff'schen Ganges und seiner Ausstülpungen oder Reste der Urnierenkanälchen, am häufigsten heterologe Wucherungen der Epithel- und Schleimhautauskleidung des Mïller'schen Ganges.) - Bezüglich der Entstehung der nicht drüsenführenden Myome wurde früher angenommen, daB sie aus den glatten Muskelfasern der Gefäßwände hervorgingen. Diese Ansicht ist jetzt fallen gelassen worden. In jüngster Zeit wurden schon bei jugendlichen Individuen Muskelzellenherde gefunden, die in der Struktur ihrer Muskelfasern von der übrigen Uterusmuskulatur abweichen. Sie werden als Ausgangspunkt der Myome angesehen. L. Fraenkel macht gelegentlich von Laparotomien, bei denen kleinere Myome enukleiert werden, auf einen ebenfalls eigenartigen Befund aufmerksam: es finden sich mitunter auf der Oberfläche des Uterus grauweiße, flache, nicht ganz scharfumschriebene und nicht enukleierbare Knötchen, von Stecknadelkopf- bis Erbsengröße. die vielleicht ein schon weiter entwickeltes Stadium der oben genannten Herde darstellen könnten.

1) Die 3. Tochter ist jetzt 11 Jahre alt und ron Herrn Prof. L. Fraenkel untersucht, jedoch kein Tumor gefunden worden. Die Annahme, daß auch sie an einem erkranken wird, die Anlage vielleicht auch schon hat, erscheint mir naheliegend. 
Von den Parovarialzysten wird angenommen, daß sie aus Resten von Drüsengängen entstehen, welche nur in der Periode des fötalen Lebens vorhanden sind.

Bei den Dermoidzysten nun dürften sich wohl alle Autoren darüber einig sein, daß ihre Entstehung auf eine Entwicklungsstörung zurückzuführen ist und daß diese Störung sehr früh eintreten muß, „wo die einzelnen Zellen noch die Fähigkeit haben, von den anderen Zellen isoliert, die Omnipotenz der Eizelle in gröBerem oder geringerem Maße ohne Befruchtung wieder zu erlangen" (Aschoff).

Die Quelle der 3 eben beschriebenen Tumoren ist demnach eine ganz verschiedene; was aber alle drei Fälle miteinander verbindet und was ihnen in gleicher Weise gemeinsam ist, das ist die Reaktion der Organismen auf irgend welche Reize (gleichviel welcher Art) mit Tumorbildung. Vielleicht muß man mit v. Rindfleisch annehmen, daß es sich um eine angeborene Schwäche der Gewebe handelt, daß die verschiedenen Reize ein primär eigenartiges, besonders geartetes Gewebe treffen, welches von Haus aus zu Lebensäußerungen disponiert ist, wie sie bei anderen Geweben nicht vorhanden sind; vielleicht aber hat Billroth mit seiner Ansicht recht, der an eine allgemeine Geschwulstdiathese dachte, erreicht durch Störungen der allgemeinen Lebensbedingungen, durch welche abnorme Stoffe von irritierender Wirkung erzeugt würden ${ }^{1}$ ). Wichtig ist jedenfalls, daß - auf diese Fälle angewendet - allen ein Moment gemeinsam ist, das man Disposition nennen möge, oder irgendwie anders, das sie jedoch zur Tumorbildung befähigt.

Nicht so klar erscheint in den eingangs erwähnten Fällen die Sachlage dadurch, daß gegen die Heranziehung der Karzinomfälle als Stütze für die ererbte Tumordisposition Einwendungen erhoben werden können. Es kann gesagt werden, daßs das Karzinom an sich ein relatir zu häufiges Ereignis im Leben aller Menschen darstelle, gemäß dem bekannten Worte: Jeder Mensch erkrankt an Karzinom, er muß nur genügend alt werden. Hierbei will ich ganz davon absehen, daß das Karzinom vielleicht nicht seltener im 4. und 5. Dezennium zur Beobachtung kommt als im höheren Alter, auch davon, daß es doch zahllose Familien gibt, in denen nicht ein einziger Karzinomfall, oder nur ganz vereinzelt einer vorkommt, obwohl die Familienmitglieder die physiologische Altersgrenze erreichen. Wollte man jedoch - grob ausgedrückt - annehmen, daß jeder Mensch im Alter sein Karzinom bekommt, dann müßten natürlich die Altersver-

1) Es wäre interessant zu erfahren, ob der Krieg mit seiner allgemeinen Unterernährung , vielleicht eine Stütze der B.'schen Hypothese geworden ist. Leider sind Beobachtungen über eine Zu- oder Abnahme der Tumoren noch nicht bekannt geworden. 
änderungen als solche als ätiologischer Faktor angesehen werden, d. h. man müBte mit Thiersch in den senilen Veränderungen des Bindegewebes die Ursache des Wachstums sehen (gedacht als der bekannte Grenzstreit zwischen Epithel and Bindegewebe). Borst ist jedoch der Ansicht, „daB die Annahme einer Veränderung der statischen Beziehungen der Gewebe zur Erklärung der Erscheinung des geschwúlstmäßigen Wachstums nicht genügt", und auch er glaubt, daß eine Störung des Gewebsgleichgewichts nur bei vorhandener Dispositon eines Gewebes zur blastomatösen Entartung für das Ausleben dieser Disposition von Bedeutung sein könne, es könnte aber nicht die Ursache des geschwulstbildenden Prozesses sein. Damit will Borst ja wohl nur sagen, daß das Alter bezüglich der Genese des Karzinoms nur als auslösender Faktor in Betracht komme bei vorhandener Disposition; d. h. das Alter würde nux dieselbe Rolle spielen, wie Entzündungserscheinungen, Traumen usw., denen die meisten Autoren ja auch nur sekundäre Bedeutung beimessen, und das wesentliche Moment wäre wieder die Disposition. Die Disposition zur Tumorbildung ist nichts Unbekanntes: Soviel ich aus der Literatur ersehen konnte, wurde diese jedoch fast immer auf genetisch gleichartige Tumoren bezogen. So beschrieb z. B. Broca eine Familie, welche in 4 Generationen 16 Krebskranke aufwies; andererseits wird auch berichtet, daß sich die hereditäre Veranlagung in manchen Fällen auf ganz bestimmte Organe erstrecke, in anderen wieder nur auf den männlichen, bzw. weiblichen Anteil der Familienmitglieder.

Wenn es mir nun gestattet wäre, aus den hier berichteten Fällen einen Schluß zu ziehen, so wäre es der, daß man annehmen müßte, es werde die Disposition zur Tumorbildung im allgemeinen vererbt, daß diese Disposition sich nicht auf eine besondere Tumorgattung, sondern auf sämtliche überhaupt vorkommenden erstrecke. Zur Geschwulstbildung wären 2 Momente Vorbedingung: 1. die Veranlagung als ursächliches. Moment, 2. die auslösende Veranlassung (Trauma, Alter, lokale Erkrankung usw.). Hier verdient eine interessante Beobachtung, die Rössle gemacht haben will, erwähnt zu werden. Er weist auf die merkwürdige Tatsache hin, daß in einzelnen Familien eine Häufung von Tumoren (am Uterus, Nieren, Magen-Darm und vielen anderen Organen) vorkamen, besonders in zunehmendem Alter, und berichtet, daß er unter 300 derartigen Fällen von benigner Neubildung zur Hälfte das gleichzeitige Vorkommen von malignen Neubildungen beobachtet habe. Er folgert daraus mit Recht auf eine Konstitutionsanomalie in den befallenen Familien, um so mehr, als sich mit den Geschwulstbildungen sehr oft auch eine Abnormität in verschiedenen Organen - in Form von Lappungen usw. vergesellschafte. Das unbekannte Moment ist wieder die "Konstitutionsanomalie"; ob sie vielleicht — wie Rössle meint — in einer krankhaften 
Schwäche des Bindegewebes besteht, ist eine Frage, die noch dringend der Aufklärung bedarf und deren Beantwortung uns ein ganzes Stück in der Therapie vorwärtsbringen dürfte. Immerhin dürfte aus den Zitaten der verschiedenen Autoren hervorgehen, daB sich die Abnormität auf den Organismus in seiner Gesamtheit bezieht, wofür ja auch die benutzten Attribute "Konstitutionsanomalie", „Disposition" sprechen. Ob Dermoidzysten erblich seien, könnte uns vielleicht, wie L. Fraenkel vorschlägt, die Wahrscheinlichkeitrechnung lehren. Es brauchte dann nur das Verhältnis gefunden $z u$ werden von familiär vorkommenden Dermoidzysten einerseits zur Summe sämtlicher Tumorbildungen, und diese Zahl müßte in Beziehung gebracht werden zu dem Prozentgehalt an Schwestern unter sämtlichen Frauen. Leider scheitert die Rechnung, da die absolute Zahl der vorkommenden Tumoren naturgemäß nicht festzustellen ist.

Immerhin wird zugegeben werdell müssen, daß das Vorkommen von Dermoidzyste bei Mutter und Tochter ein nicht unerhebliches Gewicht in die Wagschale wirft zu gunsten der Anschauunng von der Heredität dieser Tumorart, daß jedoch die Seltenheit derartiger Beobachtungen in keinem Verhältnis steht zax dem relativ häufigen Vorkommen der Dermoidzysten (in unserem Material: Dermoidzysten zu Ovarialzysten etwa wie $1: 4$ ) und daß die Frage nach der Heredität, besonders unter Berücksichtigung obiger Darlegungen, eher zu verneinen als zu bejahen ist.

Nun könnte man in den letztgenannten Fällen die Fragestellung nach der Erblichkeit der Ovarialzysten im allgemeinen erweitern, indem man die Dermoidzysten nur als eine besondere Klassifizierung der ersteren betrachtete; dann wäre allerdings das Vorkommen der beiden Tumoren im jugendlichen Alter besonders auffallend und dürfte dazu führen, eine Heredität der Zysten anzunehmen. Hierzu würden jedoch meine gesamten bisherigen Ausführungen in Widerspruch stehen; es fragt sich ferner, ob sich diese Gruppierung der Zysten aufrecht erhalten läßt, al h. ob nicht die Dermoidzysten den gewöhnlichen Zysten mindestens ihrer Genese nach zu koordinieren sind. Denn soweit ich aus der Literatur ersehen konnte, stellen sich alle Autoren auf deri Standpunkt, daß die teratoiden Geschwülste der Generationsdrüsen hinsichtlich ihrer Genese eine Sonderstellung eimnehmen, und auch Pfannenstiel betont es besonders, daß ihre Entstehung „einer besonderen Erklärung bedar"“. - - Die so häufige Kombination von Dermoiden mit Kystadenomen des Eierstocks hat sogar manche Autoren veranlaßit, die Dermoide als nichts weiter wie als Adenome des Eierstocks anzusehen (Stratz); gegen diese Auffassung wendet sich jedoch Pfannenstiel ganz entschieden und zwar in folgender Weise: ".. vielmehr ist diese Kombination ein Ausdruck dafür, daß histologisch verschiedene Bestandteile des Parenchyms gleichzeitig von demselben Reiz betroffen wurden, der die Geschwulstbildung anregt. Jeder Bestandteil 
416. Koltonski, Ueber Erblichkeit der Orarial-, besonders der Dermoidzysten.

antwortet auf diesen Reiz in der ihm eigentümlichen Weise, unter Entwicklung der ihm einmal innewohnenden formativen Tätigkeiten." Die letzten Worte dürften eine Erhärtung für meine Ausführungen darstellen; daß nämlich die Entstehung einer bestimmten Tumorart nur ein Zufallsereignis sei, abhängig von zufällig auftretenden, sie auslösenden Momenten.

So drängt alles zu der Anschauung von der angeborenen Grundlage der Geschwülste, eine Ansicht, für die auch Aschoff sich einsetzt, die zurzeit allerdings noch nicht bewiesen werden kann, deren Richtigkeit festzustellen vielleicht aber der experimentellen Tumorforschung einmal gelingen wird. Anfänge dazu finden sich jetzt bereits. Wenigstens erwähnt Aschoff selbst, daß es Mäusefamilien gibt, in welchen Spontantumoren fehlen, ferner Familien, welche für Tumorimpfung empfindlich sind oder nicht; nach seiner Ansicht deuten sich hierin hereditäre Momente an. Familien, in denen Spontantumoren fehlen, sollen auch refraktär gegen Verimpfung von Tumoren sein.

Ich glaubte, daß ein Bericht über obige Fälle erwähnenswert sei und resümiere mich kurz dahin: Es gibt eine vererbbare Tumordisposition allgemeiner Natur; die Tumorart, die im speziellen Fall zur Entwicklung kommt, stellt nur eine zufälliges Ereignis dar.

\section{Lit e r a t ur.}

A seholf, Pathologische Anatomie.

Schmaus, Pathologische Anatomie.

Biroh-Hirsobfeld, Allgemeine pathologische Anatomie.

Borst, Geschwïlste.

Rindfleisch, Gewebelehre.

Rokitanski, Pathologische Anatomie.

Ziegler, Pathologische Anatomie.

Olshausen, Die Krankheiten der Ovarien.

v. Winckel, Pathologie der weiblichen Sexualorgane.

v. Winckel, Frauenkrankheiten.

Pozzi, Gynäkologie.

Rössle, Multiple Tumoren und ibre Bedeutung. (Naturw.•med. Ges. Jena 1919.)

$\mathrm{B}$ a uer, Die könstitutionelle Disposition zu inneren Krankbeiten. Berlin 1917.

Pfannenstiel, Die Erkrankungen des Eierstocks and des Nebeneierstocks. (Veit's Handbuch.) 